Ramírez, J., Valero, G. M., Calles, R. J., Morales, A., Vera, C., y Plata, V. (2018). La formación de ciudadanos globales a través de la educación experiencial en los modelos de negociación. Revista Lebret, 10, 157-182 - ISSN Versión impresa: 2145-5996 - ISSN Versión Web on line: 2357-5468 • https://doi.org/10.15332/rl.v0i10.2202

\title{
La formación de ciudadanos globales a través de la educación experiencial en los modelos de negociación ${ }^{1}$
}

\section{The Formation of Global Citizens through the Experiential Education in Negotiation Models}

\author{
Julio Ramírez Montañez ${ }^{2}$ \\ Gladys Mireya Valero \\ Rafael de Jesús Calles ${ }^{4}$ \\ Adriana Morales Niño \\ Carlos Vera Pereira \\ Viviana Plata García
}

\begin{abstract}
Resumen
El objetivo del presente artículo fue analizar el proceso de educación experiencial desarrollado con la implementación de los modelos de negociación en escenarios reales en la Universidad Pontificia Bolivariana (Colombia), y así determinar los principales aspectos que pudieran ser considerados por las delegaciones participantes en el proceso de selección, preparación, simulación y desarrollo en este tipo de modelos. Para lograr el objetivo planteado, la metodología desarrollada se basó en el análisis de 102 encuestas realizadas a delegados de 42 universidades de 12 países que participaron en modelos de negociación en el período 2014-2018. Los resultados sugieren que los encuestados consideran que el principal beneficio de su participación en los modelos, fue la experiencia obtenida en el conocimiento del manejo de asuntos globales; así mismo, los encuestados consideran que la principal habilidad que debe tener un delegado al participar en los modelos, es saber trabajar bajo presión; de igual forma, consideran que los conocimientos adquiridos como resultado de su participación en estos modelos están relacionados con temas sobre negociación intercultural; finalmente, se determina que la principal adversidad que los delegados han enfrentado durante los modelos, es la interacción con los demás participantes debido a diferencias ideológicas, culturales e idiomáticas.
\end{abstract}

\section{Palabras clave}

Modelos de negociación, educación experiencial, National Model of United Nations, Washington Model of American States, Universidad Pontificia Bolivariana.

\section{Códigos de clasificación JEL: F02, I21, F53}

Artículo de investigación.

2 Universidad Pontificia Bolivariana. Seccional Bucaramanga. Correo electrónico: julio.ramirez@upb.edu.co. Código ORCID: 0000-0003-0116-3330

3 Universidad Pontificia Bolivariana. Seccional Bucaramanga. Correo electrónico: gladys.valero@upb.edu.co. Código ORCID: 0000-0002-5105-7512

4 Universidad Pontificia Bolivariana. Seccional Bucaramanga. Correo electrónico: rafaelcalles7@gmail.com. Código ORCID: 0000-0002-5105-7512

5 Universidad Pontificia Bolivariana. Seccional Bucaramanga. Correo electrónico: adrianam.moralesnino@ gmail.com. Código ORCID: 0000-0002-4575-2818

6 Universidad Pontificia Bolivariana. Seccional Bucaramanga. Correo electrónico: carlosvera0614@gmail.com. Código ORCID: 0000-0001-7557

7 Universidad Pontificia Bolivariana. Seccional Bucaramanga. Correo electrónico: vivianaplata97@gmail.com. Código ORCID: 0000-0002-4682-4721 


\begin{abstract}
The main purpose of this study was to analyze the process of experiential education developed with the implementation of Negotiation Models in real scenarios at Universidad Pontificia Bolivariana (Colombia) and determine the main aspects that could be considered by the delegations participating in the selection process, preparation, development and simulation in this type of models. To achieve this objective, the methodology developed was based on the analysis of 102 surveys made to delegates from 42 universities from 12 countries that have participated in negotiating models in the period 2014-2018. The results suggest that the respondents consider the main benefit from their participation in the models was the experience and knowledge gained in the management of global affairs; at the same time, respondents considered the main skill that you must have as a delegate to participate in these models, is the ability of being able to work under pressure; in the same way, they believe that the knowledge acquired as a result of their participation in these models is related to topics about cross-cultural negotiation; finally, it is determined that the main adversity that delegates have faced during the models, is the interaction with other participants due to ideological differences, cultural and idiomatic expressions.
\end{abstract}

\title{
Keywords
}

Negotiation models, experiential education, National Model of United Nations, Washington Model of American States, Pontifical Bolivarian University.

\section{Introducción}

Este artículo es resultado de una reflexión académica emprendida entre estudiantes y docentes de la Facultad de Administración de Negocios Internacionales de la Universidad Pontificia Bolivariana (Colombia), teniendo en cuenta la experiencia educativa que se ha forjado al incorporar al plan de estudios, los modelos de negociación más importantes en el ámbito internacional que se celebran en escenarios reales, como son el National Model of United Nations (NMUN) y el National Model of United Nations (WMOAS).

El National Model of United Nations (NMUN) que se lleva a cabo en la Sede Central de la ONU en Nueva York y el Washington Model of American States (WMOAS) que se conmemora cada año en la sede de la OEA en Washington, son dos de los principales referentes internacionales de programas de educación experiencial en los que grupos de estudiantes universitarios se organizan como delegaciones para desempeñar el papel de embajadores de un Estado Miembro de las Naciones Unidas o de la Organización de Estados Americanos.

Teniendo en cuenta la experiencia de la Facultad de Administración de Negocios Internacionales de la Universidad Pontificia Bolivariana al participar en el WMOAS desde 2014 y al ser la única universidad colombiana en el NMUN durante los años 2016, 2017 y 2018, los autores de este artículo identificaron la necesidad de realizar un estudio sobre los principales aspectos que pudieran ser considerados en el proceso de selección, preparación, simulación y desarrollo de los modelos de negociación en escenarios reales, debido a la poca literatura existente que aborde esta temática.

Dentro de los resultados de esta investigación se puede destacar que los delegados encuestados consideran que el principal beneficio que obtienen los participantes 
en los modelos de negociación en escenarios reales, es el aprendizaje que se genera sobre temas o problemas regionales y globales. En cuanto a las habilidades que debe tener un delegado para participar en este tipo de modelos, la mayoría de los encuestados considera que es la capacidad de trabajar bajo presión al proponer soluciones para la resolución de conflictos. Con respecto a los conocimientos que adquirieron durante los modelos, la mayoría de los encuestados señaló que los aspectos relacionados con la negociación intercultural fueron los más relevantes después de analizar su participación. De acuerdo con el principal inconveniente que tuvieron que sortear como delegados en los modelos, los encuestados coinciden en que fue el de captar la atención y el apoyo de la audiencia al momento de opinar o debatir.

Este documento se articula presentando inicialmente una caracterización de los modelos de negociación en escenarios reales, haciendo énfasis en el NMUN y el WMOAS por ser dos de los eventos académicos más destacados y de mayor tradición celebrados en escenarios reales; se presentan los antecedentes históricos de cada uno de los modelos, sus características y metodología de implementación. Luego se incluye el análisis teórico y conceptual de la educación experiencial y su aplicación con los modelos de negociación, considerando los conceptos desarrollados por Builes (2012), Toole y Toole (1995), Beraza (2011), entre otros especialistas, en este tipo de didáctica.

Posteriormente, se presentan los resultados de la encuesta aplicada a 102 delegados de diversas nacionalidades, donde se ha recopilado información relacionada con los retos y oportunidades de participar en los modelos de negociación en escenarios reales. Finalmente, se presenta una propuesta de manual que pudiera ser utilizado por futuras delegaciones en el proceso de selección, preparación y desarrollo de modelos de negociación en escenarios reales, donde se da a conocer a la comunidad científica y académica la metodología de trabajo, trayectoria, participación y demás elementos del proceso realizado dentro de las simulaciones.

\section{La formación de ciudadanos globales y los modelos de negociación en escenarios reales}

Tradicionalmente, los estudiantes de colegios y universidades aprendieron acerca de la historia y el análisis de los organismos internacionales y su funcionamiento interno a través de publicaciones impresas. En los últimos años, cursos de estudio y de información basados en Internet han sido utilizados como instrumentos útiles para analizar y aprender acerca del sistema ONU y OEA. Sin embargo, estas lecturas y estos cursos virtuales se quedan cortos en alcanzar el objetivo de dimensionar cómo se desarrollan las políticas mundiales en un entorno real.

En los últimos años ciertas disciplinas académicas han venido implementando nuevas metodologías de enseñanza y aprendizaje, las cuales pueden ser más relevantes para el mundo real en el que los estudiantes van a construir sus vidas profesiona- 
les y personales. Cada vez más, universidades de todo el mundo están incorporando el aprendizaje experiencial, que brinda a los estudiantes una experiencia práctica en la aplicación de conocimientos teóricos en la configuración real de trabajo y organizaciones (Hindriks, Jonker y Tykhonov, 2009).

Específicamente en el área de los negocios internacionales se ha venido implementando este tipo de educación experiencial con la didáctica basada en la simulación de los principales organismos internacionales, donde los estudiantes participan en juegos de rol, sirviendo como delegados de los Estados Miembros de las Naciones Unidas y de otros organismos, donde se abordan los problemas reales que enfrenta la comunidad internacional (Padierna y González, 2013). Esta propuesta de educación experiencial ha sido incorporada al ejercicio académico de la Facultad de Administración de Negocios Internacionales de la Universidad Pontificia Bolivariana con su participación en los años 2014-2018.

En estos modelos de negociación se abordan problemas globales en un contexto real. Estos modelos se desarrollan con la participación de delegaciones de distintas universidades, en los que sus representantes personifican la postura y política exterior de un Estado Miembro de la ONU u OEA en temáticas relacionadas con la agenda internacional. De igual forma, en el desarrollo de los modelos se genera un espacio pedagógico donde los participantes realizan análisis y propuestas, tomando como referencia tratados, resoluciones y mecanismos empleados dentro de los órganos de estas organizaciones, asumiendo el rol de embajador del país asignado frente al comité correspondiente.

Aunque estos modelos de negociación son simulaciones de instituciones políticas, la creciente importancia del desarrollo económico, el comercio y las cuestiones ambientales en la agenda de Naciones Unidas y OEA han creado una oportunidad para que los estudiantes de negocios internacionales se puedan enriquecer de esta experiencia académica. Los estudiantes participantes aprenden sobre asuntos globales y el papel del Estado que representa en los temas políticos, económicos, sociales y ambientales. En ese sentido, el National Model of United Nations (NMUN) y el Washington Model of American States (WMOAS) se han venido posicionando como dos de los más importantes y reconocidos modelos de negociación en escenarios reales, como los de las sedes principales de la ONU y OEA en Nueva York y Washington, respectivamente.

Según el National Collegiate Conference Association (NCCA), quien es la institución organizadora del National Model of United Nations, el principal objetivo de estos modelos de negociación es involucrar activamente a los estudiantes en asuntos globales, mediante la deliberación sobre cuestiones contemporáneas mundiales, en los mismos escenarios que son utilizados en la vida real para tales fines. Estos modelos se esfuerzan por formar ciudadanos globales que de manera civilizada desarrollan políticas multilaterales, en pos de la solución de conflictos por la vía pacífica y el desarrollo humano equitativo y sostenible (NCCA, 2017). 
Según la NCCA los fines esenciales de estos modelos son:

- Proporcionar una experiencia educativa interactiva que básicamente enseña a los estudiantes acerca de las organizaciones que se simulan.

- Ofrecer una experiencia y comprensión detallada de cómo funciona el proceso de debate internacional, la negociación y la diplomacia.

- Resolver conflictos y cuestiones planteadas originalmente en el programa de las Naciones Unidas y Organización de Estados Americanos.

- Enseña a preparar documentos, a planear una estrategia, a negociar con partidarios y adversarios, y a resolver los conflictos que afectan a casi todos los países del mundo, con el interés de la movilización de la cooperación internacional (NCCA, 2017).

\section{El National Model of United Nations (NMUN) y el Washington Model of Organization of American States (WMOAS) como principales modelos de negociación celebrados en escenarios reales}

El National Model of United Nations es el más antiguo e importante modelo de negociación internacional de todos los que se celebran en el mundo, teniendo la particularidad que es el único que se lleva a cabo en las instalaciones de la ONU en su sede Central en Nueva York. En el 2018 en la Conferencia A participaron 167 universidades de 26 países. De igual forma el Washington Model of American States (WMOAS) es el modelo de negociación más importante del hemisferio americano, el cual se desarrolla en la sede Central en la ciudad de Washington y en donde en el 2018 participaron 26 universidades, provenientes de Estados Unidos, México, Argentina, Perú y Colombia.

A continuación, se presentan los aspectos más relevantes de dichos modelos.

\section{El National Model of United Nations (NMUN)}

El Modelo Nacional de Naciones Unidas (NMUN) remonta sus orígenes al modelo intercolegial de simulación de la Liga en 1927 en la Universidad de Syracuse. Las conferencias siguientes fueron conocidas como el Modelo General de la Liga de las Naciones, las cuales fueron acogidas en diversos campus de universidades. La transición a un modelo de simulación de la ONU comenzó en 1943 en Hamilton College Campus y 1944 en Bryn Mawr College Campus, donde el modelo de las Naciones Unidas (aliados de la II Guerra Mundial) realizó simulaciones en lugar de un modelo de sociedad de las naciones (NMUN, 2017).

El NMUN promueve la comprensión de las Naciones Unidas y asuntos internacionales contemporáneos. Esta experiencia afecta positivamente las vidas de los alumnos al prepararlos para ser mejores ciudadanos globales a través de experiencias 
educativas de calidad que hacen hincapié en la colaboración y resolución cooperativa de los conflictos (UNBIS, 2018).

Es de destacar de este modelo que en algunas sesiones inaugurales del NMUN han estado presentes notables oradores a lo largo de las últimas décadas. Algunos ejemplos de NMUN como oradores principales incluyen el secretario general de la ONU, Kofi Annan en 1999; el secretario general de la ONU, Ban Ki-moon en 2008 y 2015; secretario general adjunto de la ONU, Jan Eliasson en 2013-2016; y el secretario general adjunto de la ONU, Amina J. Mohammed en 2017. Las instalaciones de la ONU, incluyendo el Salón de la Asamblea General, se utilizan comúnmente en el último día de conferencias de Nueva York. El NMUN ha sido pionero en el uso de las mismas normas utilizadas por la ONU. Las normas de procedimiento utilizadas en programas NMUN fueron adaptadas de forma real de las normas de la ONU (NMUN, 2018).

Estos son los principales NMUN que se celebran en el mundo con el auspicio de la ONU:

Tabla 1. Principales modelos MNUN en el mundo

\begin{tabular}{|c|c|c|c|}
\hline Modelo & Características & Lugar & Fecha \\
\hline NMUN-NY & $\begin{array}{l}\text { Las actividades, modelo de la ONU, } \\
\text { son programas de educación experien- } \\
\text { cial en los que grupos de estudiantes } \\
\text { están organizados como delegaciones } \\
\text { y están asignados para jugar el papel } \\
\text { de Estados Miembros de las Naciones } \\
\text { Unidas. }\end{array}$ & $\begin{array}{l}\text { NEW YORK } \\
\text {-ESTADOS } \\
\text { UNIDOS }\end{array}$ & $\begin{array}{l}18 \text { - } 22 \text { March } 2018 \\
(\text { Conference A) } \\
25 \text { - } 29 \text { March } 2018 \\
(\text { Conference B) }\end{array}$ \\
\hline NMUN - DC & $\begin{array}{l}\text { Más de } 900 \text { estudiantes universitarios } \\
\text { de todo el mundo que vienen a la ca- } \\
\text { pital de los EE. UU. y participan en } \\
\text { discusiones sobre temas que están a } \\
\text { la vanguardia de las relaciones inter- } \\
\text { nacionales. }\end{array}$ & $\begin{array}{l}\text { Washington D.C. } \\
\text { Estados Unidos }\end{array}$ & $\begin{array}{l}\text { 9-11 de noviembre } \\
\text { de } 2018\end{array}$ \\
\hline NMUN - China & $\begin{array}{l}\text { A su primera edición asistieron casi } \\
300 \text { estudiantes guiados por el ideal } \\
\text { "Un mundo, un sueño". En la simu- } \\
\text { lación realizada en } 2012 \text { se contó con } \\
\text { cuatro comités. }\end{array}$ & Xi-am, China & $\begin{array}{l}18 \text { y } 24 \text { de noviembre } \\
\text { del } 2018\end{array}$ \\
\hline $\begin{array}{l}\text { NMUN- } \\
\text { Alemania }\end{array}$ & $\begin{array}{l}\text { Promueve la comprensión de las Na- } \\
\text { ciones Unidas y los problemas inter- } \\
\text { nacionales contemporáneos. Afectará } \\
\text { positivamente las vidas de los parti- } \\
\text { cipantes y los preparará para ser me- } \\
\text { jores ciudadanos del mundo a través } \\
\text { de experiencias educativas de calidad } \\
\text { que enfatizan la colaboración y la re- } \\
\text { solución cooperativa del conflicto. }\end{array}$ & $\begin{array}{l}\text { Las sesiones se } \\
\text { llevan a cabo en } \\
\text { la Universidad de } \\
\text { Erfurt-Alemania }\end{array}$ & $\begin{array}{l}24 \text { de noviembre - } 1 \\
\text { de diciembre de } 2019\end{array}$ \\
\hline
\end{tabular}

Fuente: NMUN, 2018. Elaboración propia. 
La Facultad de Administración de Negocios Internacionales de la Universidad Pontificia Bolivariana le ha apostado a la experiencia educativa de incorporar la participación de estudiantes en el más importante modelo de negociación en escenarios reales del mundo, por la dimensión del evento, por el número de universidades participantes y por la diversidad en los países de origen, siendo la única universidad colombiana presente en el National Model of United Nations durante los años 2016, 2017 y 2018, donde 50 estudiantes de esta universidad han representado las delegaciones de Trinidad y Tobago, Colombia y Suiza. Es de destacar, los reconocimientos obtenidos en el 2018 cuando se obtuvo una mención de honor a la mejor delegación y mejor position paper en la Asamblea General 5.

\section{Washington Model of American States (WMOAS)}

Los orígenes del Washington Model of American States (WMOAS) se remontan al año 1980 como un programa conjunto de la Secretaría General de la OEA y la Universidad Georgetown, con el propósito de instruir a los estudiantes de los Estados Unidos sobre los asuntos relacionados a las Américas. El primer MOEA para universidades se llevó a cabo en Washington, D.C., del 24 al 27 de marzo de 1980. En esta Asamblea participaron diecinueve universidades del área metropolitana de Washington, D.C., Iowa, Maryland, Pennsylvania, Nueva York, Nueva Jersey, Texas, Florida, Carolina del Norte, Massachusetts y Kentucky, las cuales representaron a veintiséis Estados Miembros de la OEA. El primer MOEA para colegios secundarios se realizó en 1982 contando mayormente con la participación de colegios del área metropolitana de Washington, D.C. El programa MOEA para colegios secundarios ha crecido en alcance y prestigio y se ha venido realizando anualmente, a principios de diciembre, en la sede de la OEA (WMOAS, 2017).

En el año 1997 se llevó a cabo el primer modelo internacional para universidades realizado fuera de la sede de la OEA en Buenos Aires, Argentina. Este modelo fue auspiciado por la Universidad del Salvador y el Gobierno de Argentina. Participaron 500 estudiantes provenientes de 25 universidades de Argentina, Bolivia, Brasil, Canadá, Chile, Colombia, México, Paraguay, Uruguay y Venezuela (OEA, 1997).

A partir del 2000, además del Modelo de Washington, D.C. para universidades (WMOAS), auspiciado conjuntamente por la SG/OEA y el Instituto Interamericano de Diplomacia (IAID) y celebrado todos los años en marzo-abril, también se realizan Modelos en los Estados Miembros de la OEA. Desde el año 2009 el IAID cambió su nombre por Institute sor Diplomatic Dialogue in the Americas (IDDA) y continúa auspiciando el WMOAS.

Desde el 2005, el Programa MOEA se ha desarrollado y modernizado exitosamente, alcanzando niveles de participación sin precedentes de universidades del hemisferio (WMOAS, 2017). A continuación, se presentan los principales modelos OEA celebrados en el hemisferio americano. 
Tabla 2. Principales modelos OEA en América

\begin{tabular}{|c|c|c|c|}
\hline Modelo & Características & Fecha & Institución organizadora \\
\hline $\begin{array}{l}\text { WASHINGTON MODEL } \\
\text { OF AMERICAN STATES } \\
\text { WASHINGTON D.C. } \\
\text { ESTADOS UNIDOS }\end{array}$ & $\begin{array}{l}\text { La simulación de la Asamblea } \\
\text { General de la OEA es un even- } \\
\text { to de cinco días diseñado para } \\
\text { cumplir varios objetivos, entre } \\
\text { ellos el aprendizaje acerca de } \\
\text { las estructuras y procesos de } \\
\text { la OEA misma, aprendizaje en } \\
\text { profundidad sobre temas de ac- } \\
\text { tualidad que enfrenta el hemis- } \\
\text { ferio y un Estado miembro de } \\
\text { la OEA, y dominar el arte de la } \\
\text { diplomacia. }\end{array}$ & $\begin{array}{l}\text { Marzo } 26- \\
30,2018 \\
\text { Abril } 1-5 \text {, } \\
2019\end{array}$ & $\begin{array}{l}\text { El WMOAS cuenta con el } \\
\text { apoyo activo de la Oficina } \\
\text { del Secretario General } \\
\text { Adjunto de la OEA y ha } \\
\text { sido respaldado en una } \\
\text { Resolución de la OEA }\end{array}$ \\
\hline $\begin{array}{l}\text { MOEA - UNIVERSIDAD } \\
\text { DEL NORTE } \\
\text { UNIVERSIDAD } \\
\text { DEL NORTE - } \\
\text { BARRANQUILLA, } \\
\text { COLOMBIA }\end{array}$ & $\begin{array}{l}\text { Durante este ejercicio los estu- } \\
\text { diantes representan cada a uno } \\
\text { de los Estados Miembros de la } \\
\text { OEA y defienden las políticas } \\
\text { e intereses del país que se les } \\
\text { asigna. }\end{array}$ & $\begin{array}{l}5 \text { al } 7 \text { de } \\
\text { junio, } 2018\end{array}$ & $\begin{array}{l}\text { Este modelo está organizado } \\
\text { por la Secretaría General de } \\
\text { la Organización de los Es- } \\
\text { tados Americanos (OEA), } \\
\text { conjuntamente con la Uni- } \\
\text { versidad del Norte (UNI- } \\
\text { NORTE) }\end{array}$ \\
\hline
\end{tabular}

El modelo de la Organización de los Estados Americanos realizado en la EAFIT es un espacio en el cual los estudiantes participantes representan el rol de diplomáticos en cada una de las comisiones de la OEA, en el cual defienden la posición política e intereses de la delegación que representan por medio de debates argumentativos.

\section{MOEA - EAFIT \\ NIVERSIDAD EAFIT MEDELLÍN, COLOMBIA}

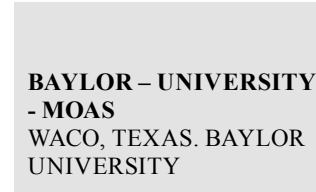

PROGRAMA MODELO DE ASAMBLEA

GENERAL DE LA OEA UNIVERSIDAD DE LA MATANZA.

BUENOS AIRES, ARGENTINA
Los delegados prenden los procedimientos parlamentarios para el debate, el caucus, cómo funcionar en grupos de trabajo de países que comparten intereses comunes y el funcionamiento de los comités que informan a la Asamblea General de la OEA.

Como ejercicio diplomático, el MOEA incluye el debate celebrado en la Asamblea General, junto con las negociaciones y el trabajo en los comités correspondientes.

Asimismo, el Modelo es el medio más efectivo del Sistema Interamericano para difundir las actividades y funciones de la OEA entre los estudiantes del hemisferio, fomentando una verdadera integración cultural en el continente.
Este evento es organizado principalmente por la Secretaría General de la Organización de los Estados Americanos (OEA) junto con la Universidad EAFIT

20 al 24 de mayo, 2018

Fuente: Elaboración propia 
La Universidad Pontificia Bolivariana ha venido participando en el WMOAS desde el 2014. En estos cinco años, cuarenta estudiantes de nuestra universidad han representado a las delegaciones de Uruguay, Trinidad y Tobago, Venezuela, Canadá y Estados Unidos, con una destacada representación de cada uno de estos Estados miembros de la Organización de Estados Americanos.

De igual manera, estos otros modelos de negociación en escenarios reales son referentes internacionales de este tipo de evento:

Tabla 3. Otros modelos de negociación

\begin{tabular}{|c|c|c|c|}
\hline MODELO & Características & LUGAR & $\begin{array}{l}\text { Institución } \\
\text { organizadora }\end{array}$ \\
\hline $\begin{array}{l}\text { Modelo Congreso } \\
\text { de la República } \\
\text { de Colombia } \\
\text { Estudiantil }\end{array}$ & $\begin{array}{l}\text { Los participantes son congresistas por un } \\
\text { espacio de dos días, a través de una simu- } \\
\text { lación donde se realizan actividades propias } \\
\text { de los miembros del Congreso de la Repú- } \\
\text { blica; el estudio y posterior debate, votación } \\
\text { de proyectos de ley que se encuentran en } \\
\text { trámite, en las Comisiones Constitucionales } \\
\text { y en Plenaria. }\end{array}$ & $\begin{array}{l}\text { Congreso de } \\
\text { la República, } \\
\text { Bogotá - } \\
\text { Colombia }\end{array}$ & $\begin{array}{l}\text { Fundación Hanns } \\
\text { Seidelm, Fundación } \\
\text { Dompaz, Unidad de } \\
\text { Atención del Ciudadano } \\
\text { del Congreso de la } \\
\text { República }\end{array}$ \\
\hline $\begin{array}{l}\text { Modelo de la } \\
\text { Organización } \\
\text { Mundial del } \\
\text { Comercio - Model } \\
\text { WTO }\end{array}$ & $\begin{array}{l}12 \text { países miembros son simulados en cada } \\
\text { edición, cada país consiste en una delega- } \\
\text { ción de } 6 \text { estudiantes, quienes están pre- } \\
\text { sentes en cada una de las } 6 \text { comisiones. } \\
\text { Como cada Comité está conformado por } \\
12 \text { personas, cada delegado será entonces } \\
\text { representante de un país distinto. En cuanto } \\
\text { a las Resoluciones, cada Comité deberá lle- } \\
\text { gar a acuerdos que sean compatibles con los } \\
\text { de los demás Comités, de esta manera, las } \\
\text { cláusulas de todos los } 6 \text { Comités se unirán } \\
\text { para formar una sola Resolución, sobre la } \\
\text { cual todos los delegados votarán el último } \\
\text { día del modelo. }\end{array}$ & $\begin{array}{l}\text { Organización } \\
\text { Mundial de } \\
\text { Comercio, } \\
\text { Ginebra - Suiza }\end{array}$ & $\begin{array}{l}\text { Universidad de San } \\
\text { Galo - Suiza, Secretaría } \\
\text { de la Organización } \\
\text { Mundial del Comercio }\end{array}$ \\
\hline $\begin{array}{l}\text { Modelo de la } \\
\text { Unión de Naciones } \\
\text { Suramericanas - } \\
\text { MUNASUR }\end{array}$ & $\begin{array}{l}\text { Los estudiantes participantes tienen la } \\
\text { oportunidad de representar a los países } \\
\text { miembros de la unión, para dar paso a una } \\
\text { simulación académica en la que se debaten } \\
\text { temas de actualidad regional, con el fin de } \\
\text { buscar un consenso mediante resoluciones } \\
\text { escritas por los mismos delegados para en- } \\
\text { contrar soluciones concretas a las diversas } \\
\text { problemáticas de la región. }\end{array}$ & $\begin{array}{l}\text { Unión de } \\
\text { Naciones } \\
\text { Suramericanas, } \\
\text { Quito - Ecuador }\end{array}$ & $\begin{array}{l}\text { Unión de Naciones } \\
\text { Suramericanas } \\
\text { (UNASUR) }\end{array}$ \\
\hline
\end{tabular}

Fuente: Elaboración propia

\section{La educación experiencial como referente teórico de los modelos de negociación en escenarios reales}

El eje temático de esta investigación es la educación experiencial enfocada a su implementación en los modelos de negociación. Builes (2000) argumenta que en un programa de educación experiencial desde el principio se estimula a las personas a 
intentar cosas que generalmente no harían ni imaginarían hacer alguna vez por sí mismos. Es decir, es una invitación a dejar esa zona cómoda y predecible que tanto nos gusta, pero en la cual ya no hay aprendizajes para entrar a una zona de riesgo, a un territorio nuevo y desconocido donde está todo por aprender y explorar.

En ese sentido, la educación experiencial es definida por Toole y Toole (1995) como "el uso de las habilidades del razonamiento crítico y creativo lo que nos ayuda a prepararnos para actuar bien y aprender de la experiencia y examinar el contexto en el que ocurre la experiencia”. De esta manera les facilita, por la naturaleza de las actividades que realizan, el conocimiento del contexto comunitario y social al tiempo que les permite brindar servicios de valor positivo en respuesta a demandas de satisfacción de necesidades externas o internas a la universidad (De Camilloni, 2017). Por lo tanto, la educación experiencial se considera como una herramienta de la pedagogía para aplicar los conceptos teóricos adquiridos por unos estudiantes en su aprendizaje y contrastarlos con la realidad, para de esta manera, adquirir vivencias que les permitan reforzar su percepción sobre el entorno para un mejor aprendizaje y capacidad de análisis.

Para ello, es necesario implementar un ciclo práctico formativo en el desarrollo educativo de los estudiantes, donde se permita abrir la caja negra, modelo donde los docentes explican sus lecciones, los estudiantes toman sus apuntes y los estudian para después reproducirlos en sus trabajos o en los exámenes (Beraza, 2011, p. 26); para permitir un conocimiento real que el estudiante adquiere con la aplicación de conocimiento teórico experimentado en un plano real. Sin embargo, deben fijarse metas y objetivos al aplicar este modelo, donde sus principios son necesarios tenerlos en cuenta para mitigar sesgos que pueden llevar al fracaso de la experimentación en planos reales.

Según Chapman, McPhee y Proudman (1992) existen una serie de principios inherentes a la educación experiencial como fundamento claro del proceso por desarrollar en las actividades proyectadas con la aplicación de esta metodología. El primer principio muestra la mezcla de contenido y proceso donde la experiencia sería insignificante al representar un sesgo respecto la teoría a estudiar y su puesta en práctica a futuro; el segundo corresponde a la ausencia de juicio excesivo por parte del facilitador permitiendo a las personas actuar bajo su propio conocimiento, entendiendo que las personas no son iguales, y cada quien es responsable de su aprendizaje; continuando con los principios, el tercero describe la manera de comprometerse en esfuerzos determinados al entender que cada quien al ser responsable de su aprendizaje se convierte en educador de sí mismo y los demás son tutores de su proceso; el cuarto fomenta la perspectiva de la generalización permitiendo una línea infinita de conocimiento y relaciones para el entendimiento situacional; el quinto principio es facilitar con estilos múltiples de aprendizaje, para ello es mejor explicarlo a través de Kolb (1976), en donde describe un ciclo de aprendizaje que incluye todos los estilos de aprendizaje: una experiencia concreta, observación reflexiva, abstraer 
conceptos y experimentar de forma activa; el sexto es el papel de la reflexión donde se debe mezclar el contenido de experiencia con las reflexiones adquiridas a través de un proceso guiado por un tutor para generar ideas coherentes; el séptimo indica crear una inversión emocional Los participantes al estar dentro de esta atmósfera de aceptación y confianza sienten que tienen un espacio para determinar su propio nivel de inversión emocional; acto seguido, se debe reexaminar los valores que se traduce en la oportunidad que conlleva al crecimiento y transformación personal; el noveno principio es la presencia de relaciones significativas con el participante, el facilitador, el medio y su entorno a partir de un proceso de aprendizaje experimentado; por último, el aprendizaje fuera de la zona de confort permite experimentar a los estudiantes actuar frente a los nuevos retos para adquirir un mayor conocimiento que a futuro será percibido como oportunidad para actuar en nuevos escenarios.

Por último, Kolb (1984) propone para su modelo de estilos preferentes cuatro fases para un proceso de aprendizaje experiencial, las cuales se clasifican de la siguiente manera:

- Observación reflexiva: en esta fase se indica cómo un individuo puede percibir a través de sus sentidos el comportamiento del entorno para llevar a cabo su desarrollo cognitivo mediante el conocimiento real de las cosas. Prácticamente sería un trabajo en campo donde el ser logra alcanzar un entendimiento real del medio para analizar las causas de los problemas de manera directa a la realidad que se percibe.

- Conceptualización: posterior a la extracción de información que obtiene el individuo en su observación reflexiva, se debe analizar las causas del porqué de las cosas para ser contrastadas con fundamentos teóricos que sustenten lo percibido, de esta manera se logra entrelazar la teoría y realidad para conocer la verdad de la situación estudiada. Es necesario dar un apoyo o tutoría al individuo en la conceptualización teórica para evitar un margen de error alto al permitir que por "sentimientos vivenciales", el individuo conceptualice desde su punto de vista con errores que podría llevar a la confusión y de esta manera al fracaso del modelo.

- Fase activa o "de rendimiento": cuando ya se cuenta con una reflexión y conceptualización, el individuo está listo para actuar en la realidad con la percepción analizada y estudiada previamente al trabajo de campo, que servirá como núcleo integrador para reforzar los conocimientos adquiridos. De este modo, la persona se encontrará en un panorama pragmático para el entendimiento propio de un objeto de estudio por analizar, donde será más fácil el entendimiento más que en un aula de clase al estilo del mito de la caverna.

- Fase, o preferencia de aprendizaje: se caracteriza por aquellos individuos que presentan una tendencia hacia el aprendizaje de manera concreta y haciendo uso de sus sentidos del tacto. 
Teniendo en cuenta lo anterior, se conceptualiza la educación experiencial como una herramienta pedagógica para aplicar en aulas de clase donde los estudiantes tendrán la oportunidad de vivir más cerca los planes de estudio por desarrollar, permitiendo enseñar con claridad temas específicos que serán replicados a futuro en el proceso de ellos. Adicionalmente, se debe otorgar la mayor cantidad de recursos posibles (tecnológicos, físicos, bibliográficos, entre otros) para permitir que el pragmatismo se convierta en vivencial, y así lograr educar desde el plano real para una enseñanza limpia y clara.

De esta manera, la aplicación de esta investigación pretende demostrar los modelos de negociación internacional como una estrategia clave de educación experiencial para el empoderamiento de los futuros líderes del mañana, ante problemáticas de tipo global en la fijación de metas que ellos alcanzarán. Por ejemplo:

La meta puede ser educativa para que los estudiantes aprendan acerca del desarrollo económico, el problema de la deuda, la reestructuración económica en los antiguos estados comunistas y socialistas, los bloques comerciales regionales, o el papel de las empresas transnacionales, los bloques comerciales, y organizaciones supranacionales en el desarrollo de los negocios internacionales, políticas, prácticas y actividades (Phillips \& Muldoon Jr., 1996).

Aunque existen variados modelos de negociación internacional de distintas OIG, el Modelo de Naciones Unidas es uno de los más representativos al recibir a casi cinco mil estudiantes de todo el mundo en dos conferencias celebradas en distintas ciudades de Estados Unidos y América Latina. Durante la conferencia, los estudiantes participan en negociaciones en grupos pequeños y grandes, donde se llevan a cabo procesos de investigación e intercambio de ideas, por medio de la búsqueda de soluciones y la redacción de documentos de posición con argumentos (UN Chronicle, 2013), lo que indica, en términos de educación experiencial, el aprendizaje en campo de un estudiante al actuar bajo un rol diplomático de alguna nación, donde analizará factores sociales, políticos, económicos y culturales de un país para tomar decisiones que pretendan un beneficio respetando las ideologías del país representado.

Adicionalmente, es la oportunidad de encaminar a los jóvenes a tener una visión global en la que se dictaminen las capacidades de los individuos a pensar de manera holística ante cualquier problemática.

En suma, para los millennials existe una opción de aprendizaje fuera del aula de clase para adquirir habilidades de pensamiento, redacción, discusión y análisis crítico mediante la práctica de negociación internacional para el mejoramiento del currículo personal y adquisición de habilidades de los estudiantes que será la ventaja comparativa frente a sus colegas en el entorno laboral. Adicionalmente, el relacionamiento con diferentes culturas internacionales se convierte en una oportunidad para el relacionamiento con fines de networking, donde se podrán eliminar barreras que motiva el etnocentrismo en la mayoría de casos. 


\section{Metodología}

La metodología implementada en la investigación es de tipo mixta, debido a que está basada en fuentes primarias y secundarias para dar respuesta a la premisa de investigación: ¿Cómo la educación experiencial influye en la formación de ciudadanos globales a través de la participación en modelos de negociación internacional?

El proceso metodológico inicia con la recolección de datos secundarios, tomando como marco teórico la educación experiencial al determinar que los modelos de negociación internacional son la práctica de las enseñanzas aplicadas por un docente en el proceso formativo de un estudiante con el ánimo de permitir una enseñanza directa en etapa vivencial, para que sea más fácil su aprendizaje y desarrollo profesional futuro. Por lo anterior, las fuentes secundarias inician con los estudios de Kolb mediante su obra El ciclo de aprendizaje experiencial. Adicionalmente, un hallazgo de datos que determina cuáles son los principales modelos de negociación que hay en el mundo y su aplicación, para finalmente servir como referencia en instituciones, especialmente de educación superior, que permitan mejorar el ciclo formativo de sus estudiantes a través del intercambio de ideas para la formación de líderes con sello internacional.

Posterior a la recolección de datos se realiza una etapa de análisis para hallar patrones que determinen la importancia de la educación experiencial, y, acto seguido, aplicar la teoría en la formulación de modelos de negociación internacional. A partir de entonces, entran en juego los datos primarios en esta investigación que se obtienen mediante la aplicación de una encuesta con las siguientes características estadísticas:

Población: Estudiantes participantes en modelos internacionales de negociación en escenarios reales.

Muestra: 102 encuestas a delegados de 42 universidades provenientes de 12 países donde se destacan 11 estudiantes de Alemania, 8 de Estados Unidos, 7 de Canadá, 4 de Italia, 3 de Francia, 2 de Venezuela, 2 de Colombia, 1 de México, 1 de Perú, 1 de Chile, 1 de Líbano y 1 de Rusia, quienes han ejercido el rol de delegados en estos modelos de negociación.

Variables: Origen de los delegados, modelo en el que participó como delegado, beneficios para los delegados, habilidades para participar en los modelos, conocimientos adquiridos durante los modelos, inconvenientes más frecuentes en los modelos.

Forma de aplicación del instrumento: Via web utilizando la plataforma de google formularios.

Teniendo en cuenta que es un modelo de negociación internacional, los encuestados son de 16 nacionalidades distintas, lo que les da una visión holística a las respuestas en términos de percepción en la cultura internacional de la educación experiencial. 
Se realizó un muestreo probabilístico, debido a que su aplicación fue a un grupo específico de personas que han participado en modelos de negociación internacional durante los últimos años, de esta manera se evitan sesgos en la investigación que podrían interrumpir el correcto curso en la recolección de datos. El formulario contiene preguntas cerradas diseñadas desde una visión general donde el encuestado logra identificar características experimentadas durante su participación como delegado en el proceso de simulación.

\section{Discusión}

En esta sección se presentan los resultados del análisis sobre la opinión y percepción de 102 participantes de los modelos de negociación internacional en escenarios reales sobre aspectos que se deben tomar en cuenta en la etapa de preparación y desarrollo de estos modelos. La tabla 3 presenta el origen de los delegados, tomando como referencia su universidad de origen. Esta encuesta fue resuelta por 102 delegados de 42 universidades provenientes de 12 países, como Colombia, México, Venezuela, Perú, Argentina, Chile, Estados Unidos, Alemania, Francia, Canadá, Líbano, Italia y Rusia.

En cuanto al modelo de negociación en el cual participó cada encuestado, de los 102 participantes, 77 fueron delegados en el National Model of United Nations, 21 en el Washington Model of American States, 1 en el Cambridge, 1 en MOVENU, 1 en Oxford y 1 en Regnum en el periodo comprendido entre los años 2014-2018.

Figura 1. ¿En qué modelo participó como delegado?

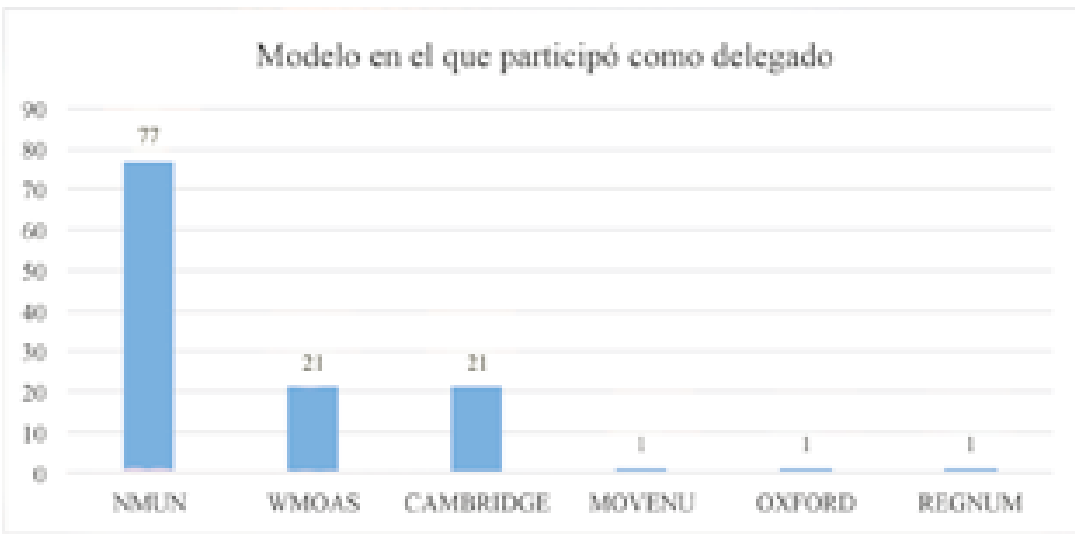

Fuente: Elaboración propia. 
Por su parte, en la figura 2 se pueden apreciar los resultados de las respuestas de los delegados encuestados, en cuanto a cuáles consideran son los principales beneficios de su participación dentro de los modelos de negociación en escenarios reales. El 27.3\% respondió que aprender más acerca de un tema o problema regional global fue el principal aporte recibido por el modelo a su formación profesional. De igual forma, el $24.2 \%$ de los delegados encuestados considera que el principal beneficio derivado de su participación en los modelos fue el mejoramiento de las habilidades para hablar en público. Finalmente, el $22 \%$ considera de vital importancia practicar negociaciones informales con estudiantes de diversas culturas.

Figura 2. Beneficios para los delegados de participar en los modelos

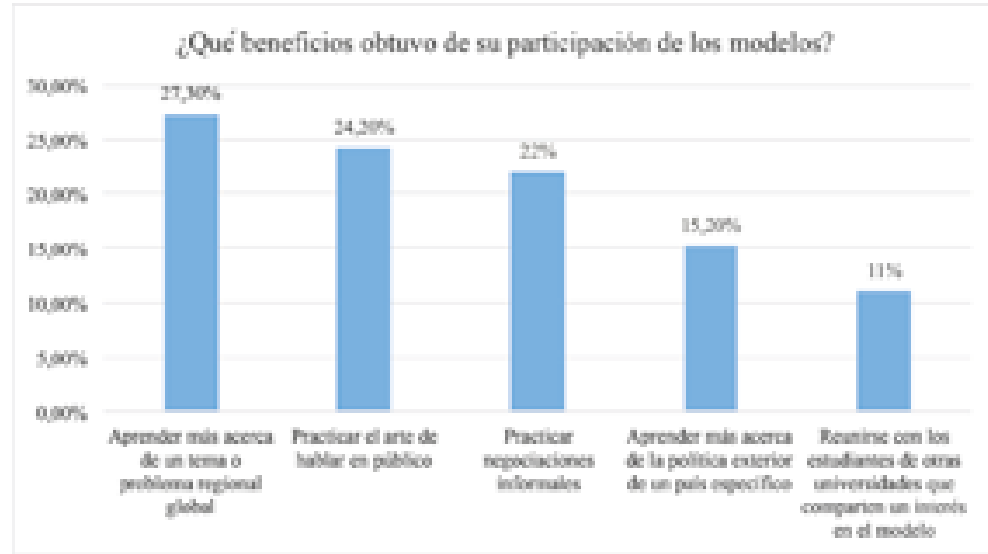

Fuente: Elaboración propia.

Teniendo en cuenta estos resultados, se puede destacar la importancia de preparar a los delegados que van a asistir a los modelos en temas de actualidad global en los campos económico, político y social. De igual forma, se infiere la importancia en la preparación a la hora de realizar discursos y cómo llevar a cabo negociaciones informales con el resto de delegados; así mismo, los encuestados señalaron cuáles son las principales habilidades que debe tener un delegado a la hora de participar en los modelos de negociación, las cuales son presentadas en la figura 3. 
Figura 3. Principales habilidades para participar en los modelos

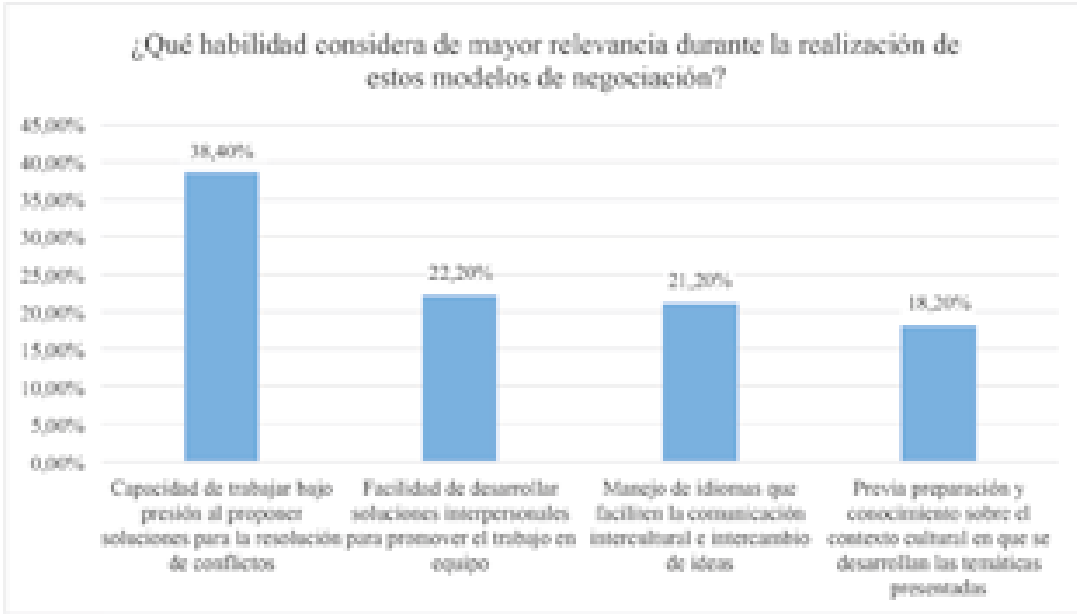

Fuente: Elaboración propia.

En cuanto a las habilidades que debe tener un delegado para participar en este tipo de modelos, el $38.4 \%$ de los encuestados considera que la principal virtud con la que deben contar los participantes en estos modelos es la capacidad de trabajar bajo presión, al proponer soluciones para la resolución de conflictos. Igualmente, el $22.2 \%$ de los delegados encuestados considera que la facilidad de desarrollar relaciones interpersonales para promover el trabajo en equipo debe ser una habilidad para tener en cuenta en el proceso de desarrollo de los modelos. Por su parte, el $21.2 \%$ de los encuestados considera que es de vital importancia contar con el manejo de idiomas que faciliten la comunicación intercultural e intercambio de ideas en estos modelos de negociación.

De los resultados de las respuestas generadas se puede inferir la importancia de conformar un equipo en cada delegación, asignando roles dentro del equipo y auditando continuamente las tareas. Esto con el fin de minimizar el choque que suele presentarse con algunos estudiantes al enfrentarse con la presión de la realidad y el alto grado de exigencia académica de los modelos. De igual forma, se debe tener en cuenta a la hora de seleccionar los delegados que estos cuenten con el manejo idiomático que les facilite la comunicación intercultural e intercambio de ideas en estos modelos de negociación.

A continuación, los encuestados señalaron cuáles fueron los principales conocimientos adquiridos durante los modelos. Las temáticas y porcentajes son presentadas en la figura 4. 
Figura 4. Conocimientos adquiridos durante los modelos

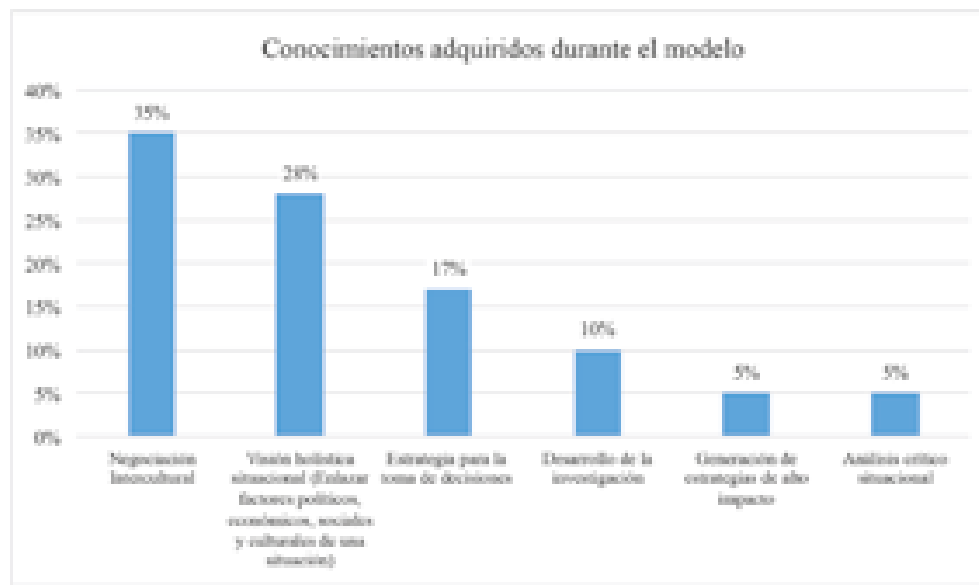

Fuente: Elaboración Propia.

El 35\% de los delegados que resolvieron la presente encuesta consideran que los conocimientos que adquirieron durante los modelos relacionados con la negociación intercultural fueron los más relevantes después de analizar su participación. Por su parte, el $28 \%$ de los delegados considera que la visión holística adquirida durante su participación en los modelos fue su principal conocimiento desarrollado. De igual forma, el $17 \%$ considera que las estrategias adquiridas para la toma de decisiones fueron el principal aporte profesional realizado por los modelos en su preparación profesional.

Estas respuestas le pueden dar una visión clara a los docentes que preparan a las delegaciones para participar en los modelos en los que se deben tener en cuenta técnicas y herramientas de negociación que les pueden ser útiles a sus estudiantes durante el desarrollo de los modelos de negociación.

Figura 5. Inconvenientes más frecuentes en los modelos

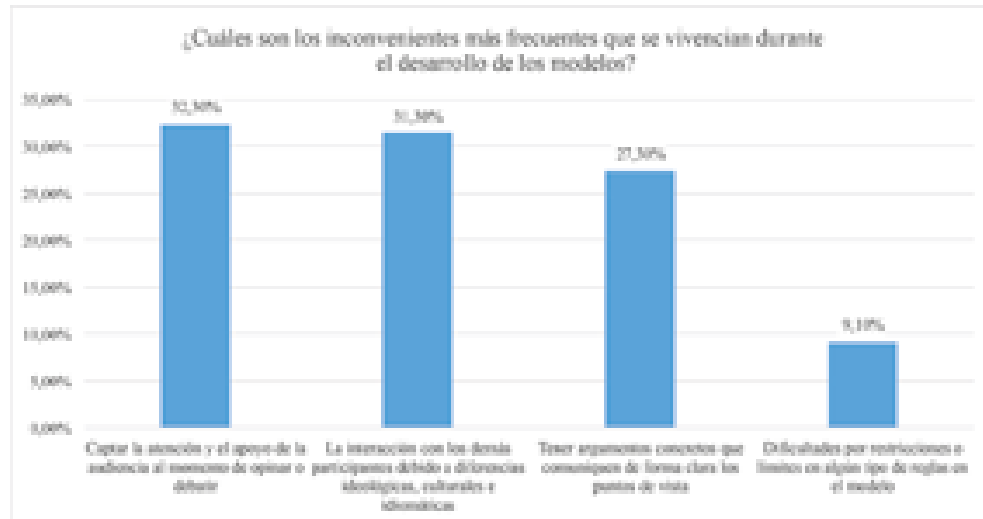

Fuente: Elaboración propia. 
El 32.3\% de los encuestados consideran que el principal inconveniente con el que tiene que sortear cada delegado durante cada modelo es captar la atención y el apoyo de la audiencia al momento de opinar o debatir. Por su parte, el 31.3\% señala que la interacción con los demás participantes debido a diferencias ideológicas, culturales e idiomáticas, es la principal barrera con la que se enfrentan los participantes. Finalmente, el $27.3 \%$ de los encuestados destaca que en algunos casos carecen de argumentos concretos que comuniquen de forma clara los puntos de vista del delegado, que puede ser un gran inconveniente para los participantes de los modelos.

\section{Manual para la selección, preparación y desarrollo de futuras delegaciones participantes en los modelos NMUN y WMOAS}

Teniendo en cuenta los resultados del instrumento de investigación aplicado a diversos delegados en modelos de negociación, los investigadores de este proyecto desarrollaron una propuesta de manual para la selección, preparación y participación en modelos de negociación en escenarios reales, el cual se espera pueda ser utilizado por estudiantes y docentes como una hoja de ruta.

Esta sección presenta una propuesta de manual de participación en los NMUN y WMOAS en los últimos cinco años. Las fases de preparación y participación en estos modelos se desarrollan en cuatro etapas: Selección de delegados, preparación de los estudiantes, simulaciones y deliberaciones, las cuales tienen una duración de ocho meses con una intensidad horaria de 4 horas semanales presenciales. El desarrollo e implementación de esta preparación se sintetiza en la tabla a continuación, y se detalla a lo largo de este capítulo.

\section{Fase de selección de delegados}

La utilización de estos modelos como una actividad curricular requiere un primer aspecto importante, como lo es la selección de los estudiantes que harán parte de las delegaciones que representarán a la universidad en estos modelos de negociación. Esta fase de selección se puede llevar a cabo mediante una convocatoria abierta a todos los estudiantes de la universidad, donde se tenga en cuenta el promedio académico, el nivel de inglés y si cuenta con VISA americana vigente, pues estos modelos se llevan a cabo en territorio norteamericano. Estos estudiantes seleccionados deben contar con unas aptitudes académicas excepcionales, las cuales deben verse reflejadas en un promedio académico superior a 3.8/5.0. De igual forma, los estudiantes escogidos deben contar con un excelente nivel de inglés para estar en capacidad de redactar resoluciones y realizar discursos ante cientos de personas en este idioma.

El examen de inglés debe ser realizado directamente por el Departamento de Lenguas de la universidad o su equivalente, donde profesionales competentes en el área realicen una prueba oral y escrita, en la que se determina el nivel de cada uno 
de los estudiantes en estas áreas y se hace una clasificación de A1 a C1. Asimismo, se recomienda que el Faculty advisor haga una entrevista en inglés, donde se busque determinar aspectos de los estudiantes, tales como su liderazgo, su capacidad de trabajo bajo presión, su capacidad de trabajar en equipo y rasgos de su personalidad que pueden ser útiles a la hora de conformar una delegación. Una vez se tenga el equipo conformado por 12 a 14 estudiantes, se inicia con las respectivas clases de preparación con una intensidad de 4 horas semanales por un periodo de 8 meses.

\section{Fase de preparación para los modelos}

Esta fase de preparación está dirigida a capacitar previamente al grupo de estudiantes en temas inherentes a la cultura, política exterior, economía y sociedad para debatir y resolver temas de tratamiento real en los órganos y comités de la ONU y OEA. Las actividades preliminares necesarias para preparar a los estudiantes para los modelos dependen de varios factores: el primero de ellos es tener un conocimiento a profundidad de la organización que se va a simular (ONU, OEA), teniendo en cuenta su estructura, su alcance, mandato y las temáticas desarrolladas en cada uno de sus órganos.

Otro aspecto importante es la capacitación en temas relacionados con los problemas internacionales. En este sentido es muy conveniente desarrollar un paquete de información para los estudiantes, donde se incluyan las normas de procedimiento de la conferencia o de simulación en el aula, información de antecedentes sobre los temas seleccionados y resoluciones de muestra y asuntos relacionados al país que se representa.

La calidad en la participación en estas conferencias depende en gran medida de la preparación de los delegados. La clave para ser una delegación exitosa en los modelos es la minuciosa y exhaustiva preparación. Por ello, se recomienda que todos los alumnos estén familiarizados con las siguientes cinco áreas de estudio:

En primer lugar, es de vital importancia realizar una investigación sobre la historia, estructura y operaciones de las Naciones Unidas u Organización de Estados Americanos. En segundo lugar, se debe desarrollar una comprensión básica de la historia, la situación socioeconómica y política, demografía, etc. de su país asignado. En tercer lugar, se debe indagar sobre el mandato, la estructura y la función de la Comisión en la cual se va a desempeñar cada delegado. A continuación, se deben investigar los temas para desarrollar en cada uno de los comités y, por último, investigar la posición del país asignado al respecto de los temas por tratar. Esta fase de preparación se lleva a cabo mediante el estudio de la estructura, historia y operaciones de la organización, el estudio del país asignado, el estudio de la comisión o comité donde se va a desempeñar el estudiante y la posición del país sobre los asuntos que tratar en cada uno de los comités. Cada uno de estos aspectos se explica a continuación: 


\section{Estudio de la estructura, historia y operaciones de la organización}

Una minuciosa preparación debe comenzar con una comprensión de las organizaciones donde se va a participar, específicamente en lo que respecta a la historia, estructura y operaciones de la organización. Cada delegado debe tener un conocimiento sólido sobre los diversos tipos de organismos dentro del sistema de las Naciones Unidas y OEA, los tipos de documentos que pueden proporcionar información sobre el tema, y los instrumentos internacionales fundamentales dentro de cada área temática, los cuales pueden ser esenciales para garantizar que las ideas expuestas en cada Comisión sean realistas.

El NMUN recomienda que cada delegado se formule estas preguntas al inicio de su preparación:

- ¿Cuáles son los distintos tipos de entidades dentro del sistema de las Naciones Unidas y del Sistema Interamericano?

- ¿Cómo actúan los diversos órganos del Sistema de las Naciones Unidas y OEA y cuáles son sus temas específicos?

- ¿Cuáles son los logros más importantes de la ONU y OEA en términos de normas y marcos jurídicos? (NMUN, 2017).

\section{Estudio del país asignado}

Una vez el estudiante esté familiarizado con la estructura, la historia y las funciones de las organizaciones por simular, el próximo paso debe ser investigar el país asignado a su universidad. Esta búsqueda de información solo se puede iniciar una vez sea asignado el país, por parte de las mesas directivas de cada modelo. Es importante en este punto investigar la estructura política, las condiciones económicas, la religión, la historia y cultura de este Estado. Puesto que todos los factores anteriormente mencionados configuran la política exterior de un Estado, la familiaridad con estas áreas le ayudará a cada delegado en la formación de una posición política coherente con respecto a su país.

En este punto es importante resaltar que el National Model of United Nations y el Washington Model of American States le dan la oportunidad a las delegaciones participantes de organizar reuniones informativas con las misiones permanentes de su país asignado ante las Naciones Unidas u OEA, las cuales son llamadas "Mission Briefings".

Estas "Mission Briefings" son muy importantes para obtener información directamente de los representantes de las misiones permanentes de los países asignados ante la ONU y OEA, las cuales resultan muy útiles en la formación de una política coherente y un estilo diplomático de acuerdo con el país representado. La experiencia en estos modelos ha demostrado que los representantes ante estas misiones perma- 
nentes suelen estar muy dispuestos a ayudar a los estudiantes, resolviendo sus inquietudes y realizando recomendaciones.

\section{Estudio de la comisión en la que se va a desempeñar cada delegado}

Con respecto a la comisión en la que se va a desempeñar cada delegado, es necesario que el estudiante comprenda su historia, gobernanza, estructura, composición, mandato, funciones y atribuciones; así mismo, tener conocimiento sobre los temas desarrollados durante los últimos períodos de sesiones y cuáles son las prioridades actuales de la comisión por representar. Una vez que la delegación tiene asignado un país, el consejero delegado decidirá cuál de los estudiantes (no más de dos por cada comisión) será asignado a cada uno de los comités.

Para tal fin, cada delegado debe comprender todos los temas del comité y deberá buscar los discursos pronunciados por su país sobre el tema y qué resoluciones se han presentado en dicha comisión. Además, se recomienda que se ponga en contacto con la sede de la comisión y solicite información específica sobre sus temas de debate, pues muchos temas en la agenda de la ONU y OEA son vastos y complejos, por lo tanto, es importante comprender cómo se desarrollan los temas en el orden del día del comité y cómo se examinan específicamente en esa comisión.

\section{Estudio de la posición del país asignado con respecto a los temas por tratar}

Para representar de forma adecuada la posición del país sobre las temáticas por desarrollarse en cada comité, es fundamental aprender y abogar por los intereses de un Estado miembro, incluso si no se está de acuerdo con la posición por parte del estudiante. Esta es la realidad de la labor de diplomáticos profesionales, que defienden los intereses de su gobierno. Esta posición será la base del documento que todos los delegados deben escribir sobre las políticas hacia los temas de la agenda. En esta fase es importante, la identificación de bloques de estados que pueden compartir las mismas perspectivas y prioridades y que pueden colaborar con el estudiante en los períodos de sesiones del comité.

Cada delegado deberá tratar de responder las siguientes preguntas:

- ¿Hay otros estados miembros u organizaciones que comparten su punto de vista?

- ¿Qué estados miembros u otras organizaciones se oponen a la posición del Estado que representa?

- ¿Cuáles estados miembros están en el mismo bloque de votantes que su estado asignado?

- ¿Está su estado asignado afiliado a alguna organización regional? 


\section{Fase de simulaciones en el aula de clases de las comisiones por representar en el NMUNy WMOAS}

Los autores del presente artículo recomiendan el desarrollo de continuas simulaciones de las sesiones con las que se enfrentarán en el modelo. Una de las formas más utilizadas por nuestras delegaciones ha sido la simulación en el aula alrededor de un tema específico. En ese sentido, el apoyo de los estudiantes que han participado en modelos anteriores ha sido útil para compartir sus experiencias con otros estudiantes en posteriores sesiones de orientación. Estas simulaciones generalmente cuentan con los siguientes pasos:

Tabla 4. Pasos en la preparación de las simulaciones en el aula de clases

\begin{tabular}{|c|c|}
\hline Paso 1: & $\begin{array}{l}\text { En la realización de la preparación de la simulación de los modelos es } \\
\text { importante establecer las metas y objetivos educativos para la simula- } \\
\text { ción. Por ejemplo, la meta educativa puede estar relacionada con que los } \\
\text { estudiantes aprendan acerca de los asuntos económicos, políticos, socia- } \\
\text { les globales, los objetivos de desarrollo sostenible, las organizaciones } \\
\text { supranacionales y la postura del país asignado dentro del modelo. Otros } \\
\text { objetivos podrían incluir el proceso de un trabajo efectivo como parte de } \\
\text { un equipo y desarrollar destrezas de comunicación escrita y oral. Una } \\
\text { vez que las metas y los objetivos han sido claramente delineados, las } \\
\text { actividades de la clase pueden ser diseñadas en consecuencia. }\end{array}$ \\
\hline Paso 2 & $\begin{array}{l}\text { Seleccionar el comité de la ONU u OEA por simular. Al tomar esta de- } \\
\text { cisión, se deben considerar los objetivos y los recursos disponibles para } \\
\text { realizar la simulación. Los recursos para este ejercicio incluyen un nú- } \\
\text { mero suficiente de estudiantes para representar a los países clave para la } \\
\text { simulación y el tiempo necesario para preparar y realizar la simulación. }\end{array}$ \\
\hline Paso 3 & $\begin{array}{l}\text { Seleccionar los temas de la agenda. Una variedad de temas pertinen- } \\
\text { tes proporcionados por la ONU y OEA pueden ser utilizados en estas } \\
\text { simulaciones. }\end{array}$ \\
\hline Paso 4 & $\begin{array}{l}\text { Establecer las normas de procedimiento para la simulación. Estas reglas } \\
\text { de procedimiento regirán las deliberaciones. }\end{array}$ \\
\hline Paso 5 & $\begin{array}{l}\text { Asignar a los países participantes. El docente debe asegurarse que los } \\
\text { países claves para este ejercicio estén incluidos. }\end{array}$ \\
\hline Paso 6 & $\begin{array}{l}\text { Preparar materiales de antecedentes que son necesarios para ayudar a } \\
\text { los estudiantes en el inicio de su investigación. }\end{array}$ \\
\hline Paso 7 & Clase de simulación. \\
\hline
\end{tabular}

Fuente: Elaboración propia.

\section{Fase de deliberaciones en Nueva York y Washington}

La fase final es el período de sesiones, este es el momento en el cual se ejecuta la simulación en la sede central de las Naciones Unidas en Nueva York y en la sede de la Organización de Estados Americanos en Washington. Es el momento en el que se ponen en manifiesto todos los conocimientos adquiridos durante la etapa anterior.

En esta parte del Modelo, cada participante debe ser capaz de desempeñar su rol de la manera más cercana posible a la realidad. Esto quiere decir, que se mantiene, 
en todo momento y con todos los participantes, un diálogo formal y respetuoso. Que cada delegación debe tratar de representar lo más fielmente posible a la verdadera, para ello puede valerse de: discursos pronunciados en sesiones de Naciones Unidas y Organización de Estados Americanos, declaraciones de los mandatarios de cada país, afirmaciones y expresiones que puedan usar los representantes de cada país.

En estas sesiones los estudiantes pasan a ocupar el lugar de embajadores de países miembros de la ONU y OEA para debatir los temas de actualidad de la amplia agenda de estas organizaciones. Los estudiantes son reconocidos como delegados del país que representan y preparan borradores de resoluciones, estrategias argumentativas, negocian con aliados y adversarios, resuelven conflictos utilizando siempre las Reglas del Procedimiento de los modelos, con la intención de movilizar la cooperación internacional para resolver los problemas que afectan a casi todos los países del planeta (Moore \& Pubantz, 2017).

Los temas de discusión de cada Comité son elegidos por la institución anfitriona, que generalmente incluye las cuestiones que se están debatiendo actualmente en las Naciones Unidas u OEA. Cada Estado miembro debe estar representado por un delegado de cada comisión. Antes del inicio del modelo, se espera que los delegados hayan llevado a cabo una exhaustiva investigación sobre sus respectivos países y tener un documento de trabajo acerca de su postura frente al tema.

Un flujo básico del proceso de deliberación en el NMUN y WMOAS es:

- La fijación de la Agenda

- Debate \& caucus

- Los documentos de trabajo

- Debate \& caucus

- Proyecto de resolución

- Enmiendas

- La votación sobre el proyecto

- Resoluciones finales

Los modelos NMUN y WMOAS utilizan reglas de procedimientos para facilitar y regular la deliberación, y pueden incluir normas generales, tales como el uso de la lengua oficial inglés, la autoridad de cada función y delegar la conducta. También contienen las normas que rigen el debate, por ejemplo, el debate de la agenda, el caucus y el procedimiento de apelación. Durante las sesiones de deliberación, los presidentes de cada comité actúan como moderadores. En algunas simulaciones, la Presidencia preparará una lista de oradores para garantizar la igualdad de oportunidad de hablar. En otros, los delegados simplemente elevan sus carteles para adquirir 
un tiempo de uso de la palabra. Sin embargo, los delegados son casi siempre responsables de intervenir solo a discreción de la Presidencia o Mesa Directiva.

\section{Conclusiones}

El presente artículo analizó el proceso de educación experiencial desarrollado con la implementación de los modelos de negociación en escenarios reales en la Universidad Pontificia Bolivariana (Colombia) y presentó una serie de aspectos que pudieran ser considerados por las delegaciones participantes en el proceso de selección, preparación, simulación y desarrollo en este tipo de modelos.

De las opiniones expresadas por los delegados en los principales modelos de negociación en escenarios reales, puede concluirse que estos perciben que el National Model of United Nations y el Washington Model of American States se han convertido en mecanismos efectivos para proporcionar a los estudiantes una experiencia internacional y multicultural importante en su formación profesional y para desempeñarse eficazmente en una economía mundial como ciudadanos globales.

Los resultados sugieren que los encuestados consideran que el principal beneficio de su participación en los modelos fue la experiencia obtenida en el conocimiento del manejo de asuntos globales; así mismo, los encuestados consideran que la principal habilidad que debe tener un delegado al participar en los modelos, es saber trabajar bajo presión; de igual forma, consideran que los conocimientos adquiridos como resultado de su participación en estos modelos están relacionados a temas sobre negociación intercultural; finalmente, se determina que la principal adversidad que los delegados han enfrentado durante los modelos, es la interacción con los demás participantes debido a diferencias ideológicas, culturales e idiomáticas.

Con las recomendaciones formuladas por los participantes del presente estudio, se diseñó una propuesta de manual de selección, preparación y desarrollo de los modelos de negociación en escenarios reales que pueda ser utilizada por futuras delegaciones. Al respecto, mientras se prepara para participar en los modelos, los estudiantes afinan una variedad de habilidades técnicas y personales. Para representar a su país con precisión, los estudiantes deben investigar minuciosamente sus países y los temas de la agenda. Para persuadir a los demás Estados miembros, estudiantes embajadores no solo deben conocer a sus países asignados, las posiciones y políticas sobre las cuestiones, sino también ejercer una escritura persuasiva, la habilidad del discurso y la capacidad de liderazgo y dotes diplomáticos. De igual forma, el proceso de preparación para estos modelos obliga a los estudiantes a aprender las habilidades más importantes de formar grupos, negociación y construcción de consenso; habilidades que también son esenciales en el entorno empresarial global. Además, los estudiantes tienen la oportunidad de interactuar con colegas de muchos países y como si fuera poco, los estudiantes también tendrán acceso a pasantías y oportunidades de prácticas en estas organizaciones internacionales. 
Finalmente, con la experiencia como participantes en este tipo de educación experiencial se encuentran considerables ventajas que obtienen los estudiantes que participan en el NMUN y WMOAS. Inicialmente, los estudiantes amplían sus conocimientos y desarrollan habilidades en el campo de las relaciones internacionales, pero además, los estudiantes que participan en estos modelos aprenden acerca de diferentes naciones y sobre la dinámica de los asuntos internacionales.

\section{Referencias}

Beraza, M. A. (2011). El Practicum en la formación universitaria: estado de la cuestión (Practicum in higher education: state of the art). Revista de Educación, $354,21-43$.

Builes Jaramillo, J. F. (2012). El abordaje de la educación experiencial. (Bachelor's thesis). Universidad de La Sabana.

De Camilloni, A. R. W. (2017). La inclusión de la educación experiencial en el currículo universitario (pp. 11-21). Universidad Nacional del Litoral. Recuperado de http://bibliotecavirtual.unl.edu.ar:8180/colecciones/handle/11185/7962

Padierna, J. C., y González, E. V. (2013). La metodología experiencial en la educación superior. Itinerario Educativo, 27(62), 151-168.

Chapman, S., McPhee, P. \& Proudman, B. (1992, agosto). What is experiential education? The Journal of Experiential Education, 15(2), 16-23.

Hindriks, K., Jonker, C. M., y Tykhonov, D. (2009, september). The benefits of opponent models in negotiation. In Proceedings of the 2009 IEEE/WIC/ACM International Joint Conference on Web. Intelligence and Intelligent Agent Technology, 2, 439-444.

Moore Jr, J. A., \& Pubantz, J. (2017). The new United Nations: International organization in the twenty-first century. Taylor \& Francis.

National Model of United Nations NMUN (2017). Delegation Preparation Guide. Recuperado de https://www.nmun.org/assets/documents/ NMUNDelegatePrepGuide.pdf

National Model of United Nations NMUN (2018). NMUN Rules and Overview. Recuperado de https://www.nmun.org/assets/documents/NMUNRules.pdf

NCCA (2017). National Collegiate Conference Association Incorporated.

Kolb, D. A. (1976). The Learning Style Inventory: Technical Manual. Boston, MA.: McBer.

Kolb, D. A. (1984). Experiential Learning: Experience As The Source Of Learning And Development. Englewood Cliffs, NJ: Prentice-Hall. 
Phillips, M. J., \& Muldoon Jr, J. P. (1996). The Model United Nations: A strategy for enhancing global business education. Journal of Education for Business, 71(3), 142-146.

Toole, J. y Toole, P. (1995). Reflection as a tool for Turning Service Experiences into learning experiences. Evaluation/Reflection, 63, 99-115.

UN Chronicle. (2013, diciembre). Teaching The UN Through Experiential Education. Recuperado de https://unchronicle.un.org/article/teaching-un-throughexperiential-education

United Nations Bibliographic Information System (UNBIS, 2018). Recuperdo de http://unbisnet.un.org/

Washington Model Organization of the American States Student Handbook. (2017). 